\title{
Excitation of vertical kink waves in a solar coronal arcade loop by a periodic driver
}

\author{
M. Selwa ${ }^{1, \star}$, K. Murawski ${ }^{2}$, S. K. Solanki ${ }^{3,4}$, and L. Ofman ${ }^{1}$ \\ 1 Department of Physics, The Catholic University of America, 620 Michigan Avenue, NE, 200 Hannan Hall, Washington, \\ DC 20064; NASA Goddard Space Flight Center, Code 671, Greenbelt, MD 20771, USA \\ e-mail: mselwa@helio.gsfc.nasa.gov \\ 2 Group of Astrophysics and Gravity Theory, Institute of Physics, UMCS, ul. Radziszewskiego 10, 20-031 Lublin, Poland \\ 3 Max-Planck-Institut für Sonnensystemforschung, Max-Planck-Str. 2, 37191 Katlenburg-Lindau, Germany \\ 4 School of Space Research, Kyung Hee University, Yongin, Gyeonggi 446-701, Korea
}

Received 29 May 2009 / Accepted 21 October 2009

ABSTRACT

\begin{abstract}
Aims. We study an oscillatory driver as a possible excitation mechanism of vertical kink loop oscillations in the ideal MHD regime. Methods. We consider a solar coronal magnetic arcade with a dense photospheric layer. The two-dimensional numerical model that we implement includes the effects of nonlinearity and line curvature on the excitation and attenuation of fast magnetosonic kink waves. We investigate the effects of a driven sinusoidal pressure pulse and compare it with the impulsive excitation by a pressure pulse that impacts the overlying loop.

Results. Our numerical simulations reveal wave signatures that are reminiscent of vertical loop oscillations seen in TRACE observational data.

Conclusions. We conclude that attenuation of vertical kink oscillations can be reduced to the value observed by adopting an oscillatory instead of an impulsive excitation. An oscillatory driver also naturally explains why only a small subset of all loops is excited to oscillate transversally in an active region.
\end{abstract}

Key words. magnetohydrodynamics (MHD) - Sun: corona - Sun: oscillations

\section{Introduction}

Sensitive instruments such as SUMER on SOHO (Wilhelm et al. 1995) and TRACE (Handy et al. 1999) have detected of various modes of oscillation in solar coronal loops. The identified wave modes include both slow propagating (De Moortel et al. 2002) and slow standing (Wang et al. 2002; 2003a,b; 2005; Ofman \& Wang 2002) waves, sausage waves (Nakariakov et al. 2003; Aschwanden et al. 2004), and two polarizations of kink oscillations called horizontal (Aschwanden et al. 1999; Nakariakov et al. 1999; Schrijver et al. 2002) and vertical (Wang \& Solanki 2004). Although these two polarizations are similar in nature, they differ in some properties (e.g., signs of compressibility are displayed by the vertical kink oscillations only). Note that some of the oscillations identified as horizontal may in fact be vertical (Wang et al. 2005).

The current work is motivated by the rapid attenuation of magnetohydrodynamic waves through leakage found by Selwa et al. $(2005,2006,2007$ a) and Gruszecki et al. $(2006,2008)$ in earlier 2D simulations. The simulated oscillations are damped considerably more rapidly than the observed oscillations. The authors modeled impulsively excited vertical kink oscillations in a solar arcade. In this paper, we propose a new excitation mechanism for vertical kink waves: an oscillatory driver instead of a single pulse. This driver can be considered as a flare that contains quasi-periodic pulsations (Ofman \& Sui 2006; Nakariakov et al. 2006; Nakariakov \& Melnikov 2009).

^ Present address: School of Mathematics and Statistics, University of St Andrews, North Haugh, St Andrews, Fife, KY16 9SS, UK
Properties of waves in 2D and 3D straight loops/slabs were described by Edwin \& Roberts $(1982,1983)$ and Roberts et al. (1984). Several papers also describe the effect of the excitation of different kinds of oscillations by a periodic driver. Murawski \& Goossens (1994) excited fast waves in a 2D slab by means of periodic photospheric shearing motions. They found that the type of excited wave (kink or sausage) depends on the polarization of the driver. Ruderman et al. (1997) studied resonant Alfvén oscillations in coronal arcades driven by toroidal footpoint motions. The authors calculated expressions for both the total amount of dissipated wave energy and its spatial distribution within the resonant magnetic surface. Tirry \& Poedts (1998) studied the heating of 2D coronal arcades by linear resonant Alfvén waves that are excited directly by photospheric toroidally polarised footpoint motions. Taroyan et al. (2005) studied the excitation of slow standing waves in a 1D loop by footpoint heat deposition. They found that the time profile of the longlasting pulse determines whether a standing or propagating wave is excited. Zaqarashvili et al. (2005) suggested that the periodic shaking of coronal magnetic field lines due to photospheric motions may induce the excitation of slow magnetoacoustic waves in short coronal magnetic structures. They found that harmonics of slow magnetoacoustic waves with half the frequency of the photospheric driver had an exponential growth in time that was proportional to the amplitude of photospheric motions and to the value of the plasma $\beta$ in the corona. Brady \& Arber (2005) studied numerically the excitation of vertical kink waves in a 2D curved loop with a velocity driver at one of its footpoints. They found that the maximum wave period, which can successfully be excited by the footpoint driving mechanism, corresponds to the fifth harmonic of the loop. In this paper, we 
extend the approaches of both Selwa et al. (2005, 2006, 2007a) and Brady \& Arber (2005) by perturbing the loop with an oscillatory pressure that acts on the whole loop from the ambient medium. Ballai et al. (2008) studied kink waves in straight cylindrical loop excited by EIT waves. The authors found that for a harmonic driver the dominant period in the generated oscillation belongs to the driver while in the case of a non-harmonic driver (modelling a shock wave) the generated oscillations in the loop are of natural periods only. This paper is devoted to a 2D MHD study. A review of 3D MHD studies of coronal loop oscillations can be found in Ofman (2009).

This paper is organized as follows. The numerical model is described in Sect. 2. The numerical results are presented in Sect. 3. This is followed by a summary and discussion of the main results in Sect. 4.

\section{Numerical model}

We describe coronal plasma by the ideal magnetohydrodynamic equations with polytropic index $\gamma=5 / 3$ :

$$
\begin{array}{r}
\frac{\partial \varrho}{\partial t}+\nabla \cdot(\varrho \boldsymbol{V})=0, \\
\frac{\partial(\varrho \boldsymbol{V})}{\partial t}+(\boldsymbol{V} \cdot \nabla) \varrho \boldsymbol{V}=-\nabla p+\frac{1}{\mu}(\nabla \times \boldsymbol{B}) \times \boldsymbol{B}, \\
\frac{\partial E}{\partial t}+\nabla \cdot\left[\left(E+p+\frac{B^{2}}{2 \mu}\right) \boldsymbol{V}-\frac{\boldsymbol{B} \boldsymbol{B} \cdot \boldsymbol{V}}{\mu}\right]=0, \\
\frac{\partial \boldsymbol{B}}{\partial t}=\nabla \times(\boldsymbol{V} \times \boldsymbol{B}), \\
\nabla \cdot \boldsymbol{B}=0 .
\end{array}
$$

Here $\mu$ is the magnetic permeability, $\varrho$ is mass density, $\boldsymbol{V}$ is flow velocity, $p$ is gas pressure, $\boldsymbol{B}$ is the magnetic field and the plasma energy density is expressed as:

$E=\frac{\varrho V^{2}}{2}+\frac{p}{\gamma-1}+\frac{B^{2}}{2 \mu}$.

Since the vertical pressure-scale height of $\sim 50 \mathrm{Mm}$ is comparable to the height of the loop $(\sim 50 \mathrm{Mm})$, the effects of gravity can be neglected to first approximation. Similarly, as our aim is to study standing fast magnetosonic waves that involve short timescales, non-ideal effects can be neglected.

\subsection{Equilibrium configurations}

We adopt and modify the coronal arcade model described in Selwa et al. (2005, 2006, 2007a). In this model, the coronal arcade is embedded in a two-dimensional motionless environment $\left(\boldsymbol{V}_{\mathrm{e}}=0\right.$; all variables referring to the environment are denoted by subscript "e") in which mass density $\varrho_{\mathrm{e}}=$ const and gas pressure $p_{\mathrm{e}}=\varrho_{\mathrm{e}} c_{\mathrm{se}}^{2} / \gamma$ has to be constant. Here $c_{\mathrm{se}}$ is the sound speed in the ambient medium. From the momentum Eq. (2), it follows that in this equilibrium configuration the Lorentz force must vanish, i.e.,

$\frac{1}{\mu}\left(\nabla \times \boldsymbol{B}_{\mathrm{e}}\right) \times \boldsymbol{B}_{\mathrm{e}}=0$.

This equation can be solved by a potential magnetic field

$\nabla \times \boldsymbol{B}_{\mathrm{e}}=0$.

Since we limit our discussion to a two-dimensional magnetically structured atmosphere for which the plasma quantities
Table 1. Equilibrium and normalization parameters for the potential arcade and the loop.

\begin{tabular}{cccccc}
\hline \hline & $\varrho\left[\frac{\mathrm{kg}}{\mathrm{m}^{3}}\right]$ & $V_{\mathrm{A}}\left[\frac{\mathrm{Mm}}{\mathrm{s}}\right]$ & $c_{s}\left[\frac{\mathrm{Mm}}{\mathrm{s}}\right]$ & $B_{0}[\mathrm{G}]$ & $T_{0}[\mathrm{MK}]$ \\
\hline Arcade & $10^{-12}$ & 1 & 0.2 & 11 & 2.9 \\
Loop & $10^{-11}$ & 0.316 & 0.2 & 11 & 0.29 \\
\hline
\end{tabular}

are independent of the spatial coordinate $y$, i.e., $\partial / \partial y=0$, the equilibrium magnetic field $\boldsymbol{B}_{\mathrm{e}}=\left[B_{\mathrm{e} x}, 0, B_{\mathrm{e} z}\right]$ has two non-zero components that are specified by the vector magnetic potential $\boldsymbol{A}=A \hat{\boldsymbol{y}}$ as

$\boldsymbol{B}_{\mathrm{e}}=\nabla A \times \hat{\boldsymbol{y}}$.

Here $\hat{\boldsymbol{y}}$ is a unit vector along the $y$-direction and $A$ satisfies Laplace's equation, $\nabla^{2} A=0$ with its solution

$A(x, z)=B_{0} \Lambda_{B} \cos \left(x / \Lambda_{B}\right) \mathrm{e}^{-z / \Lambda_{B}}$.

This method leads to the equilibrium magnetic field components

$B_{\mathrm{e} x}=B_{0} \cos \left(x / \Lambda_{B}\right) \mathrm{e}^{-z / \Lambda_{\mathrm{B}}}$,

$B_{\mathrm{e} z}=-B_{0} \sin \left(x / \Lambda_{B}\right) \mathrm{e}^{-z / \Lambda_{B}}$

where $B_{0}$ is the magnetic field at the level $z=0$ and $\Lambda_{B}$ is the magnetic scale height such that

$\Lambda_{B}=\frac{2 L}{\pi}$

Here $L$ is the horizontal half-width of the arcade. In this model, the Alfvén speed in the environment is given by $V_{\mathrm{Ae}}=$ $\left|B_{\mathrm{e}}\right| / \sqrt{\mu \varrho_{\mathrm{e}}}$.

Following Selwa et al. (2005, 2006, 2007a) we model a denser loop that is embedded in the arcade in such a way that its edges follow two specific magnetic field lines. We choose $x_{\mathrm{f}}= \pm 0.7 \mathrm{~L}$ as footpoints of the loop and $w=0.0125 \mathrm{~L}$ as its half-width at the footpoints. Such a loop does not have a perfect circular shape, but its average radius and length can be estimated to be $70 \mathrm{Mm}$ and $190 \mathrm{Mm}$, respectively. The mass density is enhanced in the loop compared to the ambient medium. Initially a top-hat density profile is introduced into the loop. For the mass density contrast, we choose $d=\varrho_{\mathrm{i}} / \varrho_{\mathrm{e}}=10$, where $\varrho_{\mathrm{i}}$ denotes the mass density within the loop and $\varrho_{\mathrm{e}}$ corresponds to the ambient medium. This density contrast was chosen to remain consistent with our earlier computations (Selwa et al. 2005, 2006, 2007a). Note that the Alfvén speed within the loop is smaller than in the ambient medium $\left(V_{\mathrm{Ai}}=V_{\mathrm{Ae}} / \sqrt{d}\right)$. As a consequence, the loop forms a wave guide for fast magnetosonic waves (as described in Edwin \& Roberts 1982). Because of the decrease in magnetic field strength with height and the constant pressure loop, the plasma $\beta=2 c_{\mathrm{s}}{ }^{2} /\left(\gamma V_{\mathrm{A}}{ }^{2}\right)$ grows from 0.048 at the loop footpoints to 0.125 at the loop apex and the loop is cooler than the surrounding corona. It may affect slow waves evolution as their speed is lower inside the loop than in the outer part of arcade. The influence of the sound speed on fast magnetosonic speed is minor in low $\beta$ plasma, and therefore the lower loop temperature is not expected to affect our results significantly. Recently Ugarte-Urra et al. (2009) found that cooler loops, e.g., at transition region temperatures are observed in active regions (mainly peripheral parts) and reach heights of $150 \mathrm{Mm}$ in the corona above the limb. All equilibrium parameters are listed in Table 1. 


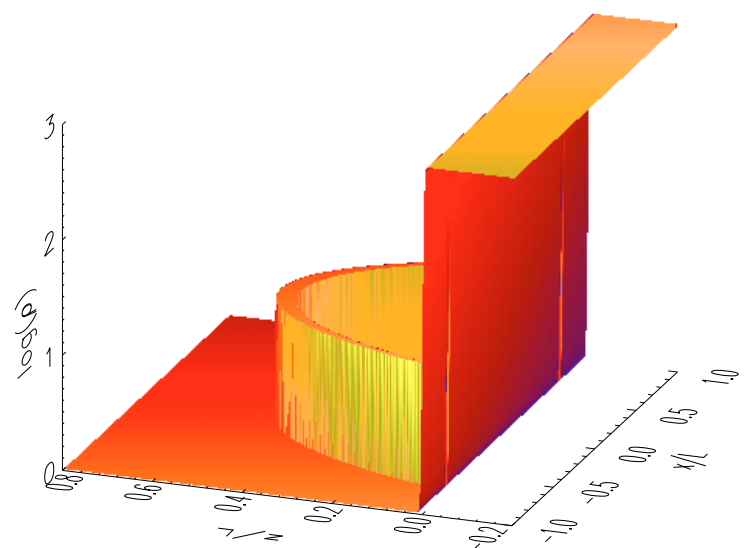

Fig. 1. Initial mass density profile. Note the logarithmic scale on the vertical axis.

\section{Numerical simulations}

We adopt the numerical code Athena developed by Gardiner \& Stone (2005). This code implements a higher-order Godunov method for solving time-dependent ideal MHD equations. This algorithm, which is based on the piecewise parabolic method, is second-order accurate in space and time. Equation (5) is satisfied by using a constrained transport scheme.

Equations (1)-(5) are solved numerically in an Eulerian box with the $x$ - and $z$-dimensions $(-L, L) \times(-0.2 L, 2.8 L)$. This box is covered by a uniform grid of $400 \times 600$ numerical cells. Grid convergence studies have shown that this resolution is sufficient for obtaining results independent of the grid.

We apply open boundary conditions with a zero-gradient extrapolation of all plasma variables at all the boundaries to allow a wave signal to freely leave the simulation region. Instead of adopting the line-tying boundary conditions at the bottom of the simulation region implemented by Selwa et al. (2005, 2006, 2007a), we model wave reflection from the bottom boundary by implementing a dense photosphere-like layer for $z<0$ region given by

$\varrho_{0}(z)=\varrho_{\mathrm{e}}\left\{1+\frac{d_{\mathrm{ph}}-1}{2}\left[1-\tanh \left(\frac{z}{s_{\mathrm{ph}}}\right)\right]\right\}$.

Here $d_{\mathrm{ph}}=\varrho_{\mathrm{ph}} / \varrho_{\mathrm{e}}=1000$ is the photospheric mass density contrast and $s_{\mathrm{ph}}=2.5 \mathrm{Mm}$ enables the smoothness of the density profile to be controlled. The equilibrium structure of the loop and the photospheric layer is shown in Fig. 1.

\subsection{Perturbations}

To excite the oscillations, we implement a damped oscillatory driver in gas pressure given by

$\delta p(x, z, t)=A_{\mathrm{o}} p_{\mathrm{e}} S(x, z) S(t)$,

where

$$
\begin{aligned}
S(x, z) & =\exp \left[-\left(\frac{x-x_{\mathrm{o}}}{w}\right)^{2}\right] \exp \left[-\left(\frac{z-z_{\mathrm{o}}}{w}\right)^{2}\right], \\
S(t) & =\frac{1}{2}\left[1+\cos \left(\frac{2 \pi}{P_{\mathrm{o}}} t\right)\right] \exp \left(-\frac{t}{\tau_{\mathrm{o}}}\right) .
\end{aligned}
$$

Here $A_{\mathrm{o}}$ denotes the amplitude of the driver, whose initial position is denoted by $\left(x_{0}, z_{0}\right)$ and whose width is given by $w$. Symbols $P_{\mathrm{o}}$ and $\tau_{\mathrm{o}}$ are the wave period and attenuation time of

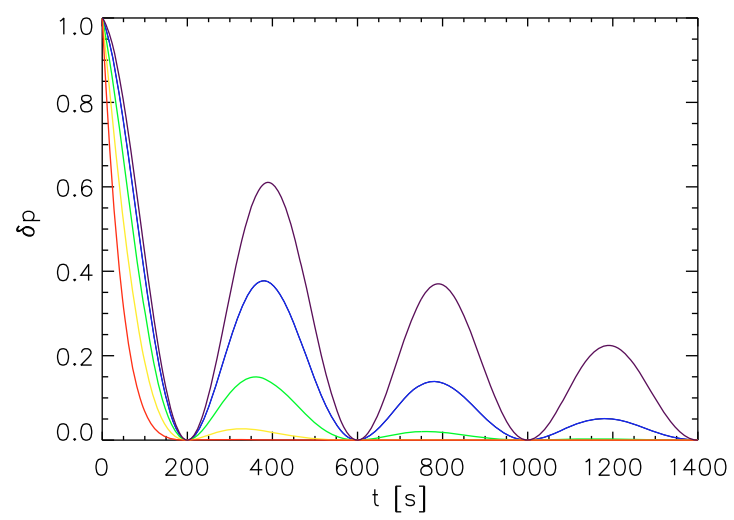

Fig. 2. Several time signatures of an oscillator with wave period $P_{\mathrm{o}}=$ $400 \mathrm{~s}$ and $\tau_{\mathrm{o}}=50 \mathrm{~s}$ (red line), $\tau_{\mathrm{o}}=100 \mathrm{~s}$ (yellow line), $\tau_{\mathrm{o}}=200 \mathrm{~s}$ (green line), $\tau_{\mathrm{o}}=400 \mathrm{~s}$ (blue line), and $\tau_{\mathrm{o}}=800 \mathrm{~s}$ (violet line). The initial amplitude of the perturbation is normalized to unity.

Table 2. Impulse over unit area ${ }^{1}$ equivalent to the oscillator with parameters $A_{\mathrm{o}}=0.025, P_{\mathrm{o}}=400 \mathrm{~s}$.

\begin{tabular}{cc}
\hline \hline$\tau_{\mathrm{o}}[\mathrm{s}]$ & $\delta p t$ \\
\hline 50 & 1.01 \\
100 & 1.49 \\
200 & 1.89 \\
400 & 2.17 \\
800 & 2.32 \\
\hline
\end{tabular}

the pulse, respectively. We choose $x_{0}=0, z_{0}=0.25 \mathrm{~L}$, and $w=0.25$ L. Selwa et al. (2006) studied the influence of the position and width of a single pulse on the excitation, attenuation, and other properties of oscillations. Note that spatial parameters are fixed for both kinds of excitation. Several examples of time signatures of an oscillatory driver are shown in Fig. 2, where we plot drivers with different attenuation times. All have amplitudes, $\delta p$, normalized to unity and $P_{\mathrm{o}}=400 \mathrm{~s}$. Table 2 shows the pulse amplitude, $A_{\mathrm{p}}$, corresponding to the oscillator with parameters $A_{\mathrm{o}}=0.025$, and $P_{\mathrm{o}}=400 \mathrm{~s}$ for different $\tau_{\mathrm{o}}$. Here we assume that the driver acts within the time interval $0<t<200 \mathrm{~s}$. This time interval corresponds to the time before the driver reaches its first minimum, which occurs before the loop oscillations display their first maximum. As a result, this part of the driver can act like a single pulse if the attenuation is sufficiently rapid.

To be able to compare with the results of Selwa et al. (2005, 2006, 2007a), we also perturb the static solution of Eqs. (1)-(5) by launching a hot pulse in the gas pressure, i.e.,

$\delta p(x, z, t=0)=A_{\mathrm{p}} p_{\mathrm{e}} S(x, z)$,

where $A_{\mathrm{p}}$ is the relative amplitude of the pulse.

\subsection{Results}

We begin our studies by launching an initial pulse in gas pressure according to Eq. (18). Since this case will be used for comparison purposes with excitation by an oscillatory driver, we choose an amplitude that provides a comparable integral over time as an osillatory driver until the first minimum in its time signature: $A_{\mathrm{p}}=2.5$ (see Table 2). Next we excite oscillations by an oscillatory driver (Eq. (15)). As a reference value, we choose

\footnotetext{
${ }^{1}$ Calculated to be the area beneath the curve within the time interval $0<t<200 \mathrm{~s}$
} 

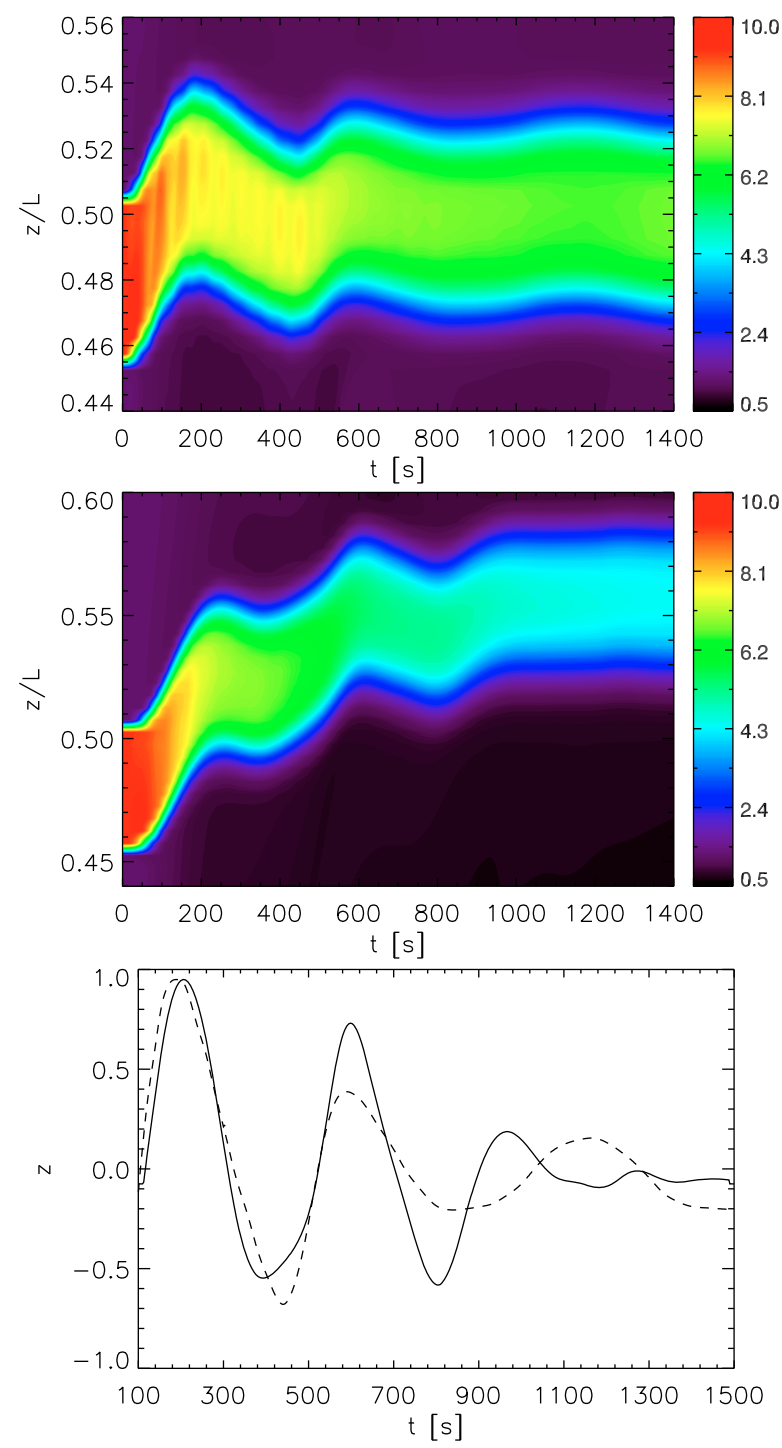

Fig. 3. Time-signatures of the mass density (color scale; units of $\varrho_{\mathrm{e}}$ ) at the loop apex. Oscillations are excited by a pulse (top panel) and a damped oscillatory driver (middle panel). Spatial coordinates and time are measured in units of $L$ and seconds, respectively. Bottom panel shows time signatures of the mass density at the loop's apex after removing trends from the top (dashed line) and middle (solid line) panel, respectively.

$A_{\mathrm{o}}=0.025, P_{\mathrm{o}}=\tau_{\mathrm{o}}=400 \mathrm{~s}$. Figure 3 shows the time signatures of mass density collected at the loop apex for impulsive (top panel) and oscillatory (middle panel) excitation, respectively. The oscillations excited by the oscillatory driver (middle panel) are less attenuated than those produced by impulsive excitation (top panel) as we demonstrate by means of the analysis in this section (although the difference is not clearly visible from Fig. 3).

To identify the excited mode of oscillations, we plot difference images of the loop between the initial state $(t=0)$ and the first maximum observed in the time series of loop apex height $(t=250 \mathrm{~s})$ (top panel of Fig. 4) and between the first maximum $(t=250 \mathrm{~s})$ and the following minimum $(t=400 \mathrm{~s})$ (bottom panel of Fig. 4). We clearly see that the fundamental mode of the vertical kink oscillation is excited. This matches both the observational data (compare with Fig. 3 in Wang \& Solanki 2004) and impulsively excited oscillations (compare with Fig. 2 in Selwa et al. 2005). Local enhancements (white patches), seen among
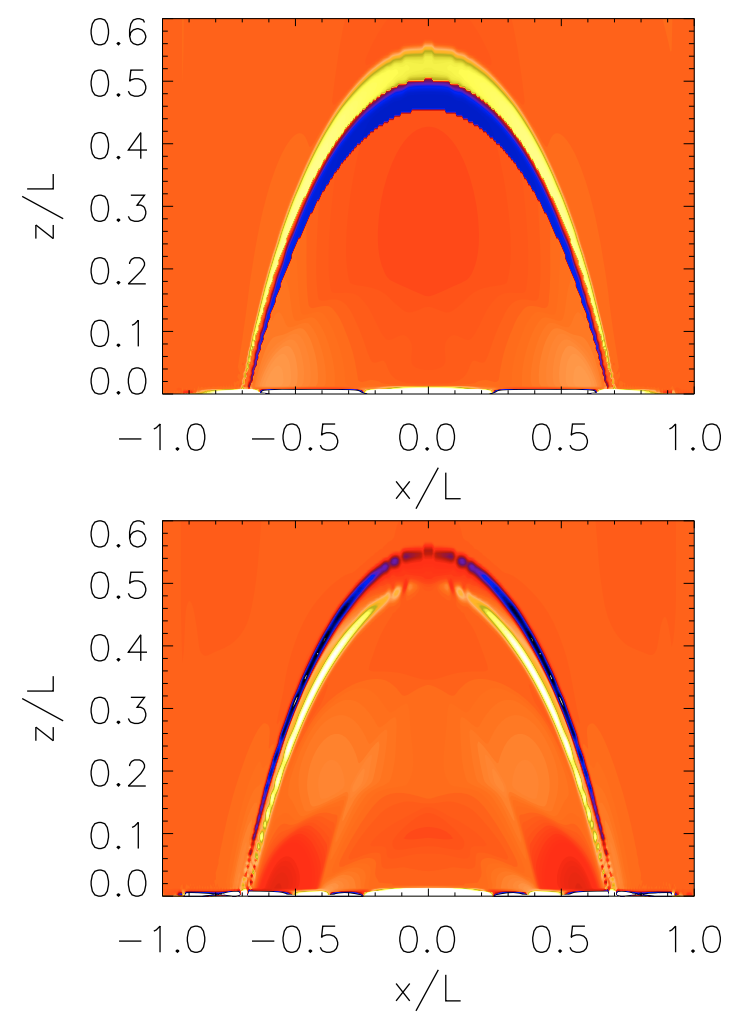

Fig. 4. Difference images of the loop in the case of oscillatory excitation $\left(A_{\mathrm{o}}=0.025, P_{\mathrm{o}}=\tau_{\mathrm{o}}=400 \mathrm{~s}\right)$. Top panel corresponds to the difference between initial state and the first maximum of the oscillation (bottom panel of Fig. 3), bottom panel shows the difference between the first maximum in time and the following minimum. Yellow indicates a positive difference (i.e., the new position of the loop) while the blue colour corresponds to a negative difference, i.e., with respect to the loop at an earlier time.

yellow arcs indicating the new position in time of the loop in the lower panel, correspond to slow magnetosonic waves that propagate along the legs of the loop (Selwa et al. 2006). These waves are produced by magnetic compression of the loop that also leads to the evacuation of part of the mass from the apex, which can be seen in both panels of Fig. 3 and the bottom panel of Fig. 4. At a later stage of the evolution, the mass density at the loop's apex is again enhanced (not shown) after the reflection of the slow waves from the footpoints of the loop - this process leads to the formation of a slow standing wave (Selwa et al. 2007b). The other feature that amplifies the effect of mass rarefication at the apex is numerical diffusion discussed qualitatively and quantitatively by Selwa et al. (2006, 2007a).

We trace the global oscillations of the loop discussed above by following the position of the loop apex in time estimated by the maximum of a Gaussian function fitted to the mass density profile across the loop's cross-section at its apex

$G(z)=G_{0} \cdot \exp \left[-\frac{\left(z-G_{1}\right)^{2}}{2 G_{2}^{2}}\right]+G_{3}$

where $G_{1}$ denotes the position of the loop apex, $G_{0}+G_{3}$ is the amplitude of the Gaussian function (mass density at the loop apex), and $G_{2}$ is the half-width of the Gaussian function (halfwidth of the loop). Before carrying out any further analysis, we removed any long term trend by smoothing the time signature to obtain pure oscillations of the apex position versus time. Then 


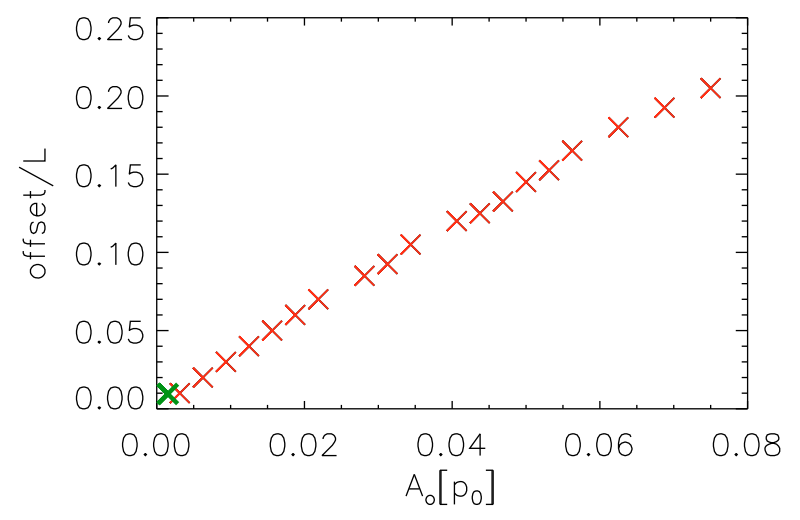

Fig. 5. Offset of the loop's apex (measured in units of $L$ ) versus amplitude of the oscillator with $P_{\mathrm{o}}=\tau_{\mathrm{o}}=400 \mathrm{~s}$. Thick green symbol corresponds to excitation by a pulse.

we fitted a damped sine function to constrain the wave periods and attenuation times of the oscillations:

$D(t)=D_{0}+D_{1} \cdot \sin \left(\frac{2 \pi}{P} t+D_{2}\right) \exp (-t / \tau)$,

where $P$ denotes the wave period of the oscillation and $\tau$ is the attenuation time. The offset of the loop's apex resulting in a longterm trend strongly depends on the strength of the driver (i.e., $A_{\mathrm{o}}$ shown in Fig. 5).

We begin our parametric studies with an impulsively excited wave. The fitting described by Eq. (20) provides $P_{\mathrm{p}}=424$ (440) $\mathrm{s}$ and $\tau_{\mathrm{p}} / P_{\mathrm{p}}=1.03(0.97)$ for a fit to part of the oscillation time series containing 2 (3) maxima (top panel of Fig. 3). The subscript $p$ identifies the quantities related to the excitation by the pulse. Although this wave period is about two times longer than the observed $\sim 230$ s period of the displacement oscillations (Wang \& Solanki 2004), it can be reduced to the exact observational value with a different choice of the Alfvén speed, $V_{\mathrm{Ae}}$, in the corona, which is a free parameter in our model. However, the attenuation time is approximately three times shorter than the observed value of $\tau / P \sim 3$ (Wang \& Solanki 2004), irrespective of the choice of Alfvén speed, $V_{\mathrm{Ae}}$. The dimensionless ratio $\tau / P$ is expected to be independent of $V_{\mathrm{Ae}}$ in the linear regime. Parameters such as the amplitude or width of the pulse also influence the wave period and attenuation time: e.g., a narrower and smaller amplitude pulse results in shorter wave periods and less attenuated oscillations (Selwa et al. 2006), which, however are still attenuated too quickly. The inferred period is close to the kink period of the loop, which in $2 \mathrm{D}$ is given by

$P_{k}=\frac{2 l}{V_{\mathrm{Ae}}}$

where $l$ is the loop length. The choice of external Alfvén speed for calculation of the period is not so straightforward. Since the equilibrium magnetic field exponentially decreases with height, we decided to estimate the period with the mean value of Alfvén speed outside the loop, e.g., $\left(V_{\mathrm{Ae}_{\text {max }}}+V_{\mathrm{Ae}_{\text {min }}}\right) / 2$, where $V_{\mathrm{Ae}_{\text {max }}}$ and $V_{\mathrm{Ae}_{\text {min }}}$ are maximum/minimum values of Alfvén speed around the loop. This estimation infers the period $\sim 515 \mathrm{~s}$. This value is $\sim 20 \%$ higher than the derived value. However, from Fig. 4 we know that the observed mode is a vertical kink mode. A difference in period produced by averaging in the estimated external Alfvén speed, may be caused by the complex geometry of the system (curved loop with non-uniform cross-section) and the effect of including a photospheric boundary instead of line-tying at the coronal boundary of the loop footpoints.
The top panel of Fig. 6 displays the wave period $P$ versus the relative amplitude of a driver $A_{\mathrm{o}}$ (for $P_{\mathrm{o}}=\tau_{\mathrm{o}}=400 \mathrm{~s}$ ) in the case of a fit based on 2 (squares) or 3 (crosses) maxima in the corresponding time signature (middle panel of Fig. 3). In Figs. 6-8, points corresponding to impulsive excitation are plotted in green. They are shown for comparison purposes. We clearly see that for a low amplitude driver, the wave period of the oscillations is close to the wave period of the oscillation excited impulsively, $P_{\mathrm{p}}$, because only the first peak of the driver is significant enough to excite oscillations. For a larger driver amplitude, the wave period of the oscillations tends toward the wave period of the driver $\left(P_{\mathrm{o}}=400 \mathrm{~s}\right)$. Up to $A_{\mathrm{o}}=0.04$, the trends based on 2 and 3 maxima fits in $P\left(A_{\mathrm{o}}\right)$ are consistent. However, for larger amplitudes they exhibit the converse behavior, because a stronger pulse produces a higher shift of the loop apex and the entire loop is more stretched so that its length changes significantly. As a consequence of that, the distance between the loop and the driver increases resulting in a decrease in the observed wave frequency (increase in wave period) between the first and the second maxima in time signatures. In this way, we observe the influence of the Doppler effect. Because the loop is shifted mainly between the 1st and the 2nd maximum, the Doppler effect influence is visible mainly for the fit with 2 maxima (squares). We estimated analytically the change in observed period, $\delta P$, caused by the Doppler effect

$P+\delta P=\frac{V_{\mathrm{A}}+V_{\mathrm{s}}}{V_{\mathrm{A}}} P$,

where $V_{\mathrm{s}}=\frac{\Delta L}{\Delta t}$ is the velocity at which the loop recedes from the driver, measured from the shift of the apex of the loop, $\Delta L$, in time, $\Delta t$. The periods calculated from Eq. (22) agree with values shown in Fig. 6 with squares for $P \rightarrow P_{\mathrm{o}}$. The fit based on 3 maxima (crosses) is much less affected by the "Doppler effect", because after the second maximum the position of the apex is almost fixed (bottom panel of Fig. 3) and the Doppler effect becomes very small.

As shown by Selwa et al. (2007a), a major attenuation mechanism of vertical kink oscillations in a 2D ideal MHD model is energy leakage. It was found that energy leakage causes attenuation to increase for longer period oscillations. Since the excitation mechanism does not change the properties of the plasma, we expect that the attenuation mechanism should be the same for both impulsive and oscillatory excitation. Indeed, the middle and bottom panels of Fig. 6 show the attenuation time measured in units of wave period, $\tau / P$, versus the relative amplitude of the driver, $A_{\mathrm{o}}$, and versus wave period, $P$. It is clear that the normalized attenuation time, $\tau / P$, falls off for longer period oscillations. This agrees with the findings of Selwa et al. (2007a) for the energy leakage mechanism (compare with the top panel of their Fig. 8). We plot only crosses in this panel to avoid the results being too strongly affected by the Doppler effect. Note the high $\tau / P$ values reached for large $A_{\mathrm{o}}$.

Next we hold the amplitude and attenuation time of the driver fixed: $A_{\mathrm{o}}=0.025, \tau_{\mathrm{o}}=100 \mathrm{~s}$, and vary its period $P_{\mathrm{o}}$. Figure 7 displays the wave period $P$ and attenuation ratio $\tau / P$ of the oscillations versus the period of the oscillator. For this value of $\tau_{\mathrm{o}}$, we expect the oscillatory driver to behave like a single, long-lasting pulse that is almost independent of $P_{\mathrm{o}}$ except at the shortest periods (e.g., red and yellow curves in Fig. 2). From the top panel of Fig. 7, we see that a short-period strongly attenuated driver excites oscillations with wave periods close to the value obtained by an impulsive excitation (green). As the period of the oscillator increases, we observe a small change (decreasing trend) in the periods of the oscillations $(<10 \% P)$. If $P_{\mathrm{o}} / \tau_{\mathrm{o}} \leq 1$, then the 
A\&A 512, A76 (2010)
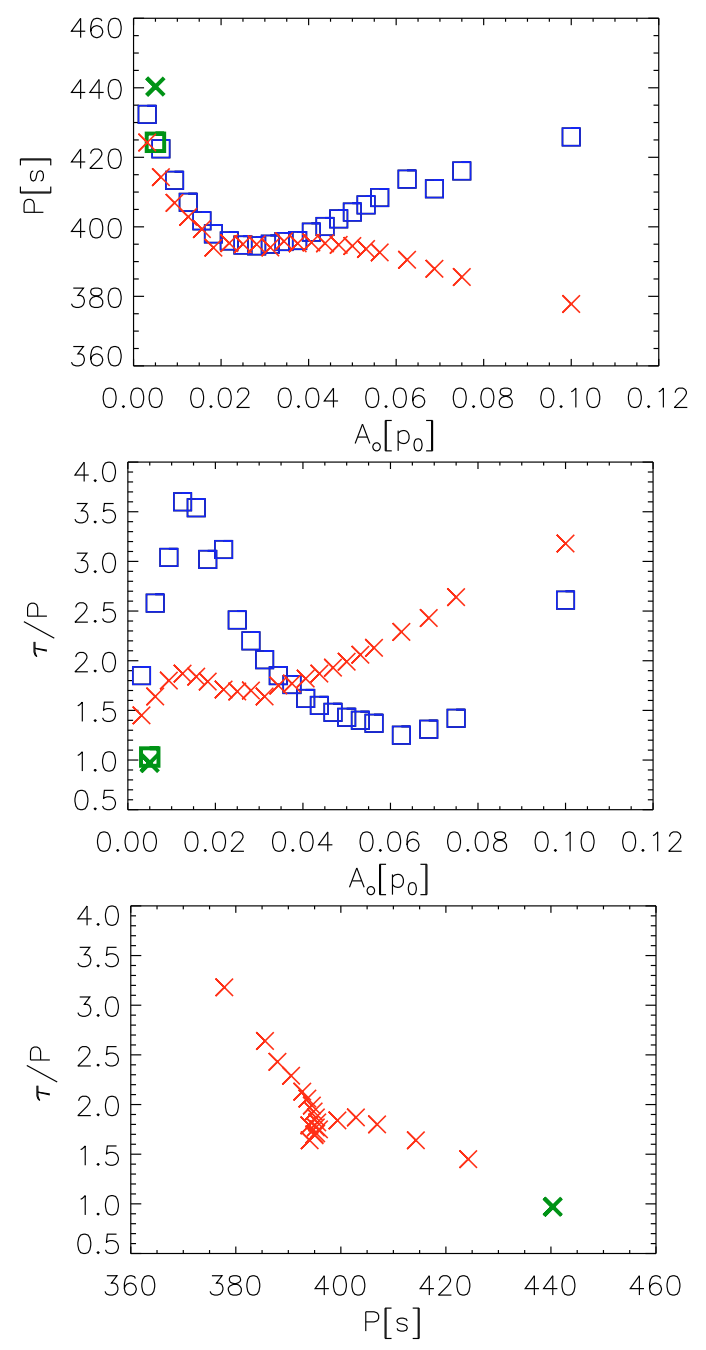

Fig. 6. Wave period, $P$, (top panel) and the ratio of attenuation time $\tau$ to wave period, $\tau / P$, (middle panel) of vertical loop oscillations versus amplitude of the oscillator with $P_{\mathrm{o}}=\tau_{\mathrm{o}}=400 \mathrm{~s}$. Bottom panel displays the normalized attenuation time $\tau / P$ versus wave period of the oscillations, $P$. Thick green symbols correspond to excitation by a pulse. Squares (crosses) correspond to damped sine function fits including 2 (3) significant maxima of the damped oscillations.

driver acts like a real oscillator (note the high $\tau / P$ value in the bottom panel of Fig. 7 for small $P_{\mathrm{o}}$ ). However, as $P_{\mathrm{o}}$ increases we indeed find a long-lasting single pulse and $\tau / P$ drops to the value expected for a pulse excitation. Note that the driver periods were chosen to be close to a fraction or a multiple of the loop's eigenperiod. Drivers with periods that do not fulfill this criterion (e.g. $300 \mathrm{~s}$ ) do not excite an eigenmode and give rise to extremely strongly damped oscillations.

Next we vary the attenuation time of the oscillator with its amplitude $\left(A_{\mathrm{o}}=0.025\right)$ and period $\left(P_{\mathrm{o}}=400 \mathrm{~s}\right)$ fixed. The top panel of Fig. 8 shows yet again that the strongly attenuated oscillator $\left(\tau_{\mathrm{o}} \ll P_{p}\right)$ acts like a single pulse and both the wave period of the excited oscillation and its attenuation time tend to the values for an impulsive excitation (green color). For higher values of the attenuation time of the oscillator (less attenuated driver), the wave period of the excited oscillation tends to the period of the driver, $P_{\mathrm{o}}$. The middle panel of Fig. 8 shows that the oscillations are less attenuated for a less attenuated driver. The steady increase in $\tau / P$ with $\tau_{\mathrm{o}}$ is particulary striking. The bottom panel of Fig. 8 shows a similar trend as the bottom panel of Fig. 6, which again agrees with the findings of Selwa et al. (2007a, their
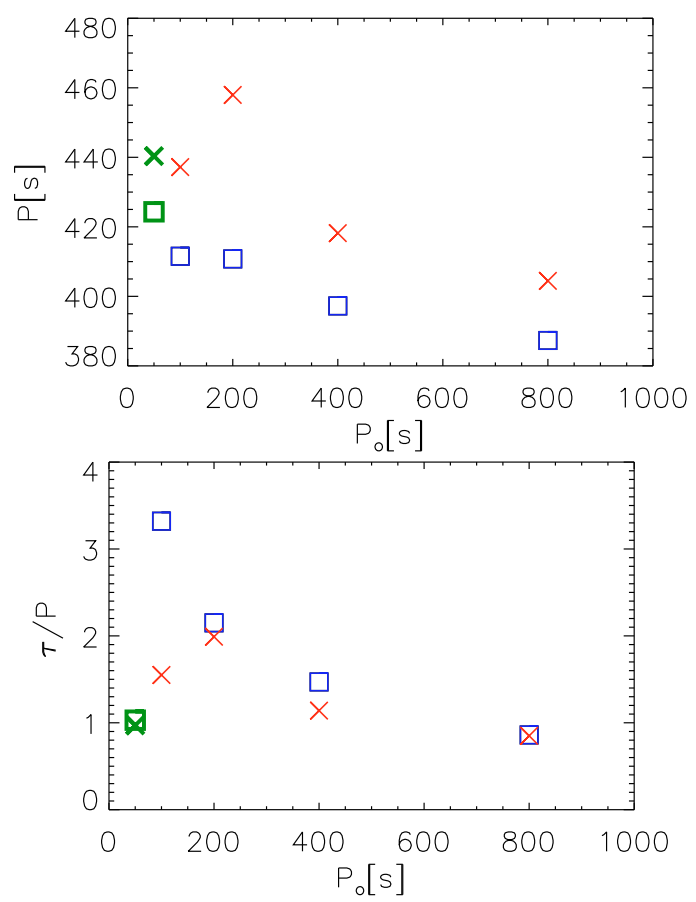

Fig. 7. Wave period, $P$, (top panel) and the ratio of attenuation time $\tau$ to wave period, $\tau / P$, (bottom panel) of vertical loop oscillations versus period of the oscillator with amplitude $A_{\mathrm{o}}=0.025$ and attenuation time $\tau_{\mathrm{o}}=100 \mathrm{~s}$. Symbols are the same as in Fig. 6.

Fig. 8) that energy leakage is a viable attenuation mechanism for vertical kink oscillations.

\section{Summary}

We have carried out the first study of vertical kink oscillations in a 2D curved loop excited by an oscillatory driver outside the loop. This driver could model a shock with a wave train behind it excited by a flare. Our numerical results show that the oscillations excited by the attenuated periodic driver are in general less attenuated than those excited by a pulse. The ratio of $\tau / P$ becomes close to the observational value $(\tau / P \sim 3$, Wang $\&$ Solanki 2004) for short-period slowly attenuated drivers. Impulsive excitation results in rapid attenuation $(\tau / P \sim 1)$, similar to the excitation by a footpoint driver in velocity studied by Brady \& Arber (2005), who calculated the decay time for the fundamental mode ( 0.18 wave periods) based on an extrapolation from higher harmonics. We find that the agreement of the damping time to period ratio between our model and observation is an encouraging result, suggestive of the form of the excitation mechanism of vertical oscillations observed in coronal loops. On the other hand, impulsive excitation results in a time signature that is closer to the observations (top panel of Fig. 2 in Wang \& Solanki 2004) since the time-signature of the loop apex of the oscillations excited by the oscillator displays a strong upward shift (Fig. 3). This is an artifact of the chosen expression for the driver (Eq. (15)), which gives perturbations that are entirely positive (i.e., only pressure enhancements) and results in a higher shift of the apex due to positive flux of energy into the system. We realize that this driver may approximate poorly the conditions in the solar corona, but we wish to avoid setting negative pressures in the code, which could otherwise arise for largeamplitude drivers. In excitation by a pulse, the eigenoscillation is set up naturally in a coronal loop. A restriction of a driven 
M. Selwa et al.: Excitation of vertical kink waves by a periodic diver
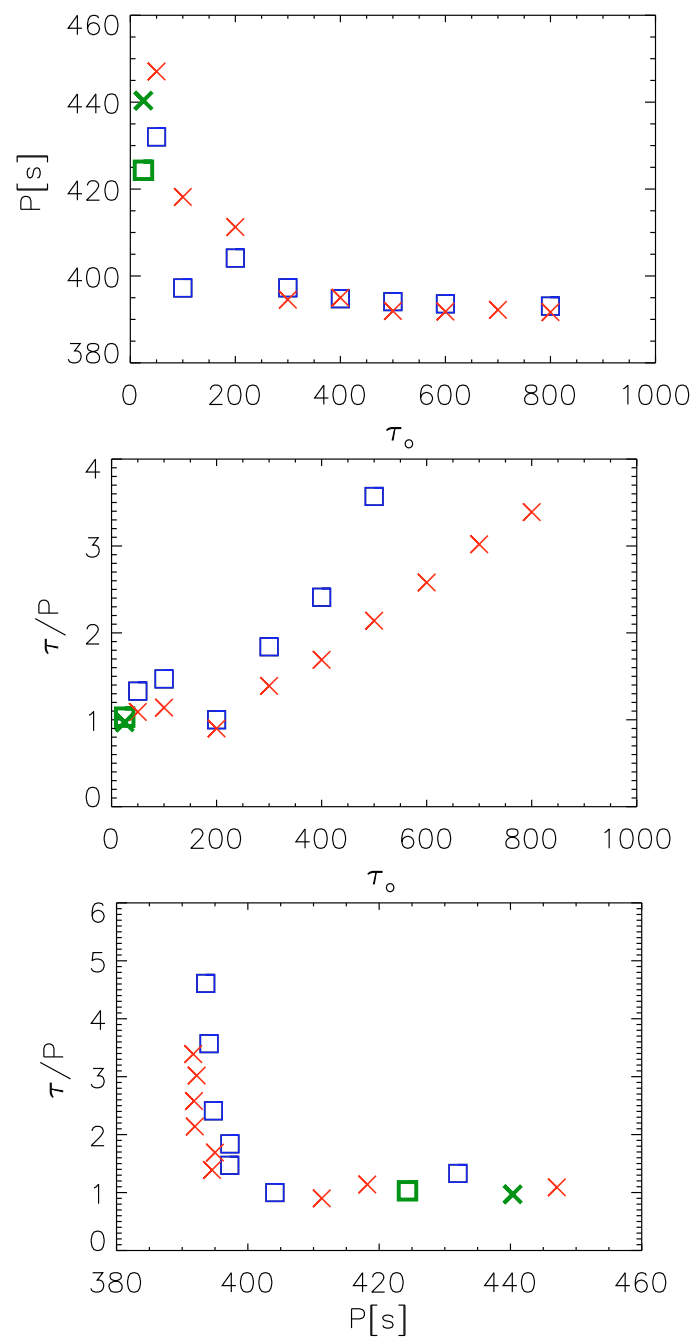

Fig. 8. Wave period, $P$, (top panel) and the ratio of attenuation time $\tau$ to wave period, $\tau / P$, (middle panel) of vertical loop oscillations versus attenuation time of the oscillator with $P_{\mathrm{o}}=400 \mathrm{~s}$. Bottom panel displays the normalized attenuation time $\tau / P$ versus wave period of the oscillations, $P$. Symbols are the same as in Fig. 6.

system is that only if a driver period is close to the eigenperiod it generates an eigenoscillation.

Typically, an external oscillatory driver with a given frequency in an active region will be able to excite transverse oscillations only in a small subset of loops, namely those whose eigenfrequencies are close to the frequency of the driver. This can explain why only very few loops of an active region are excited to oscillate transversally after an energetic event such as a flare. An impulsive driver, which harbours a very wide range of frequencies, should excite a much larger fraction of all loops.

We also showed that a rapidly damped oscillator acts like a single pulse. By varying the period of the driver it is possible to excite oscillations that resemble a fundamental vertical kink mode, but which are just a very strongly attenuated simple response of the loop to a periodic driver. In addition, our results are consistent with the findings of Selwa et al. (2007a) that vertical kink oscillations are attenuated because of energy leakage.

In this study, we did not change the properties of the loop, such as the spectrum of eigenfrequencies, $F(\omega)$, by switching between impulsive excitation and the excitation by the damped oscillatory driver. However, the observed change in decay rates (and periods) can be explained in terms of changing the convolution $F(\omega) * R(\omega)$, where $R(\omega)$ is the response function of the loop for the excitation mechanism. Although the oscillator is characterized by a single wave-period $P_{\mathrm{o}}$, it corresponds to a more complex Fourier spectrum. This is partly related to the decay of its amplitude with time and also its spatial structure. So, the oscillator launches a continuous spectrum of wave-periods centered around $P_{\mathrm{o}}$. If $P_{\mathrm{o}}$ fits the eigenperiod of the loop, $P_{\mathrm{p}}$, then the excited oscillations will be magnified by the oscillator. On the other hand, in the case of a mismatch between $P_{\mathrm{o}}$ and $P_{\mathrm{p}}$, loop oscillations receive signals from waves produced because the oscillator is out of phase (at unsuitable moments of time). As a result, loop oscillations are incoherent to the oscillator and the loop exhibits more complex oscillations, which are more attenuated.

In the applied model, we have neglected several important factors such as the three-dimensional geometries of loops and the flare site, radiative losses, and gravitational stratification. A $2 \mathrm{D}$ model is more apropriate to the excitation of vertical oscillations than other transverse oscillation modes, since the source of the initial pulse must be located in or near the loop plane. However, the absence of the third dimension may result in higher leakage, especially with initial top-hat loop density profile becoming diffused with time, and smooth loop edges being another factor that increases the leakage.

One possible extension of our model is to study more realistic drivers such as with random parameters $\left(A_{\mathrm{o}}\right.$ or $\left.\tau_{\mathrm{o}}\right)$. A possible way of reducing the offset is to replace the pressure driver by a vertical (or perpendicular to the loop) velocity driver, which, according to Gruszecki et al. (2008, private communication), results in a lower offset because it is not associated with an internal energy (slow waves).

Acknowledgements. M.S. thanks Dr. Tongjiang Wang for his comments. The magnetohydrodynamics code used in this study was developed at Princeton University by Tom Gardiner, Jim Stone, Peter Teuben and John Hawley with support of the NSF Information Technology Research (ITR) program. M.S.'s \& L.O.'s work was financially supported by the NASA SEC Theory program and NASA grant NNG06GI55G. K.M.'s work was supported by a grant from the State Committee for Scientific Research Republic of Poland, with MNiN grant for years 2007-2010. S.K.S.'s work has been partially supported by the WCU grant No. R31-10016 funded by the Korean Ministry of Education, Science and Technology.

\section{References}

Aschwanden, M., Fletcher, L., Schrijver, C., et al. 1999, ApJ, 520, 880 Aschwanden, M., Nakariakov, V., \& Melnikov, V. F. 2004, ApJ, 600, 458 Ballai, I., Douglas, M., \& Marcu, A. 2008, A\&A, 488, 1125

Brady, C. S., \& Arber, T. D. 2005, A\&A, 438, 733

De Moortel, I., Ireland, J., Walsh, R. W., et al. 2002, Sol. Phys., 209, 61

Edwin, P. M., \& Roberts, B. 1982, Sol. Phys., 76, 239

Edwin, P. M., \& Roberts, B. 1983, Sol. Phys., 88, 179

Gardiner, T. A., \& Stone, J. M. 2005, J. Comput. Phys., 205, 509

Gruszecki, M., Murawski, K., Selwa, M., et al. 2006, A\&A, 460, 887

Gruszecki, M., Murawski, K., \& McLaughlin, J. 2008, A\&A, 489, 413

Handy, B. N., Tarbell, T. D., Wolfson, C. J., Korendyke, C. M., \& Vourlidas, A. 1999, Sol. Phys., 190, 351

Murawski, K., \& Goossens, M. 1994, A\&A, 286, 952

Nakariakov, V., \& Melnikov, V. 2009, Space Sci. Rev., DOI: 10.1007/S11214009-9536-3

Nakariakov, V. M., Ofman, L., Deluca, E. E., Roberts, B., \& Davila, J. M. 1999, Science, 285, 5429, 862

Nakariakov, V. M., Melnikov, V. F., \& Reznikova, V. E. 2003, A\&A, 412, 7

Nakariakov, V. M., Foullon, C., Verwichte, E. \& Young, N. P. 2006, A\&A, 452, 343

Ofman, L., \& Wang, T. J. 2002, ApJ, 580, L85

Ofman, L., \& Sui, L. 2006, ApJ, 644, L1490

Ofman, L. 2009, Sp. Sci. Rev., 149, 153

Roberts, B., Edwin, P. M., \& Benz, A. O. 1984, ApJ, 279, 857

Ruderman, M. S., Goossens, M., Ballester, J. L., et al. 1997, A\&A, 328, 361

Schrijver, C. J., Aschwanden, M. J., \& Title, A. M. 2002, Sol. Phys., 206, 69 
A\&A 512, A76 (2010)

Selwa, M., Murawski, K., Solanki, S. K., Wang, T. J., \& Tóth, G. 2005, A\&A, 440,385

Selwa, M., Solanki, S. K., Murawski, K., Wang, T. J., \& Shumlak, U. 2006, A\&A, 454, 653

Selwa, M., Murawski, K., Solanki, S. K., et al. 2007a, A\&A, 462, 1127

Selwa, M., Ofman, L., \& Murawski, K. 2007b, ApJ, 668, L83

Smith, J. M., Roberts, B., \& Oliver, R. 1997, A\&A, 317, 752

Taroyan, Y., Erdélyi, R., Doyle, J. G., et al. 2005, A\&A 438, 713

Tirry, W. J., \& Poedts, S. 1998, A\&A, 329, 754

Ugarte-Urra, I., Warren, H. P., \& Brooks, D. H. 2009, ApJ, 695, 642
Wang, T. J., Solanki, S. K., Curdt, W., Innes, D. E., \& Dammash, I. E. 2002, ApJ, 574, L101

Wang, T. J., Solanki, S. K., Curdt, W., et al. 2003a, A\&A, 406, 1105

Wang, T. J., Solanki, S. K., Innes, D. E., Curdt, W., \& Marsch, E. 2003b, A\&A, 402, L17

Wang, T. J., \& Solanki, S. K. 2004, A\&A, 421, L33

Wang, T. J., Solanki, S. K., Innes, D. E., et al. 2005, A\&A, 435, 753

Wang, T. J., Solanki, S. K., \& Selwa, M. 2008, A\&A, 489, 1307

Wilhelm, K., Curdt, W., Marsch, E., et al. 1995, Sol. Phys., 162, 189

Zaqarashvili, T. V., Oliver, R., \& Ballester, J. L. 2005, A\&A, 433, 357 